\title{
A simple student laboratory practice for the study of light scattering by cylindrical bodies
}

Toto Mabiala Masiala, Edmond Phuku Phuati, B. Albert Kazadi Mukenga

Toto Mabiala Masiala, Edmond Phuku Phuati, B. Albert Kazadi Mukenga, "A simple student laboratory practice for the study of light scattering by cylindrical bodies," Proc. SPIE 10452, 14th Conference on Education and Training in Optics and Photonics: ETOP 2017, 104522 U (16 August 2017); doi: 10.1117/12.2269915

SPIE Event: 14th Conference on Education and Training in Optics and Photonics, ETOP 2017, 2017, Hangzhou, China 


\title{
A simple student laboratory practice in the study of light scattering by cylindrical bodies.
}

\author{
Mabiala Masiala,Toto ${ }^{(a)}$,Phuku Phuati,Edmond ${ }^{(a)}$,Kazadi Mukenga B. Albert ${ }^{(a)}$ \\ (a) Physics department, University of Kinshasa, BP190 Kinshasa, Democratic Republic of \\ Congo
}

\begin{abstract}
The study of light scattering by cylindrical bodies is of great importance in many aspects, but most of the time; it is studied in a very theoretical manner. In that work, we present a simple manner to study scattering of light by cylindrical bodies. The method combines the use of a simple experimental set-up using a He-Ne laser as a source, a circular paper screen, and the use of a simple code for simulation using the Lorentz-Mie formalism. In that way, the student can compare the experimental results with the simulation. They can qualitatively notice the difference of the behavior of scattering by a low-loss dielectric cylinder, a metallic cylinder, and an absorbing cylinder. Simulation can help students to follow the evolution of the scattering regime when the relative diameter of the cylinder, with respect to the incident wavelength, changes. A focus is stressed especially when the radius becomes far below the wavelength.
\end{abstract}

Keywords: nano optics, light scattering, backscattering, sub wavelength scattering

\section{INTRODUCTION}

Cylinders are common geometries that are found in the design of many devices ${ }^{(1)}$ such as antennas, resonators and optics fibers. Moreover many natural structures can be modeled as $\quad$ cylinders ${ }^{(2)}$ (biological bodies, ice clouds...). The study of light scattering by such structures is of great importance in many aspects. Actually with the advent of nano-sciences it has become important to study the behavior of such geometries when their dimensions reach or become smaller than the incident wavelength. In the other hand it is also important to study the evolution of scattering regime when the dimensions of the scattering cylinder are very large in comparison to the wavelength of the incident radiation. Actually cylindrical photonic structures (Bragg mirrors) plays an important role from plasmonic, to textile fibers physical coloration; so the importance of a progressive approach in teaching the behavior of homogenous cylinder is important before the study of multicoated cylinders.

14th Conference on Education and Training in Optics and Photonics: ETOP 2017, edited by Xu Liu,

Xi-Cheng Zhang, Proc. of SPIE Vol. 10452, 104522U · ( 2017 ICO, IEEE, OSA, SPIE

CCC code: $0277-786 X / 17 / \$ 18$ - doi: 10.1117/12.2269915

Proc. of SPIE Vol. $10452104522 \mathrm{U}-1$ 


\section{METHODS}

\subsection{Theory and simulation}

The theory used for computing our simulation is based on the formalism developed by Mie for bodies of spherical shapes (3) and later developed for cylindrical geometries ${ }^{(4)(5)}$. The theory gives a rigorous solution of the Maxwell equations for a plane monochromatic wave impinging a cylinder under any incidence with respect to the cylinder axis. The far-field solution for the scattered field is given by:

The expression of the far field scattered intensity for an infinite cylinder under normal incidence is finally given in the form:

For the case I:

$$
I_{11}=\frac{I_{0}}{\pi k R}\left|b_{01}+2 \sum_{n} b_{n 1} \cos (n \theta)\right|^{2}
$$

For the case II:

$$
I_{22}=\frac{I_{0}}{\pi k R}\left|a_{02}+2 \sum_{n} a_{n 2} \cos (n \theta)\right|^{2}
$$

Where $a_{n 2}$ and $b_{n 1}$ are the Mie coefficients for the scattered field. The lower indices (1) and (2) in the coefficients refer to cases I ( $\vec{E}$ parallel to the cylinder axis) and to case II ( $\vec{E}$ orthogonal to cylinder axis).

They are given in the form:

$$
\begin{aligned}
& a_{n 2}=\frac{m J_{n}(y) J_{n}^{\prime}(x)-J_{n}(x) J_{n}^{\prime}(y)}{m J_{n}(y) H_{n}^{2^{\prime}}(x)-H_{n}^{2}(x) J_{n}^{\prime}(y)} \\
& b_{n 1}=\frac{J_{n}(y) J_{n}^{\prime}(x)-m J_{n}(x) J_{n}^{\prime}(y)}{J_{n}(y) H_{n}^{1 \prime}(x)-m H_{n}^{1}(x) J_{n}^{\prime}(y)}
\end{aligned}
$$

(m) is the relative refractive index of the scattering material, $J_{n}$ is the cylindrical Bessel function of first kind, $H_{n}^{2}$ is the Hankel function of the second kind. (x) is the size parameter and (y) is the product of the refractive index with the size parameter.

Knowing these elements, the far-field intensity distribution can be computed using a simulation code using MATLAB ${ }^{\circledR}$, has been performed using that model on a simple PC. Input parameters are the incident wavelength of the source; the corresponding refractive of the material at the selected wavelength, and the radius of the cylinder. Number of terms of the series for computations has been limited to: $n_{\max } \approx x+4.3 x^{1 / 3}{ }^{(6)}$. Where $\quad(x)$ is the size parameter. Computations have been performed for three different types of materials say a high loss dielectric (silicon monoxide), metal (nickel), and low-loss dielectric (fused silica). Radius range from very large cylinders (size parameters up to 5000), to very thin cylinders (size parameters as low as 0.1 ). 


\subsection{Experiment}

Experiments have been conducted using a linearly polarized He-Ne source emitting at $632.8 \mathrm{~nm}$. The polarization orientation has been determined using a detector, and a polarizer which plane is orthogonal with the horizontal plane. It is placed through the path of the Laser source; than Laser is rotated until the detector shows a maximum in the intensity received in the detector. This means that the direction of the polarizer and that of the source coincides. Experiments is then performed for the field parallel to cylinder axis and the source is rotated $90^{\circ}$ to make the field orthogonal to cylinder axis.

The first experiment is didactical using a circular paper screen, graded in degrees, perforated to allow the beam go through the screen (figure1). The target is placed on the centre of the circle, with a radius of $60 \mathrm{~cm}$ to satisfy the far-field condition. The cylinder is placed vertically with respect to the horizontal plane. The scattering pattern is displayed on the screen. A special attention is made for forward and backscattering. Figures show the schematic of the experiments.

The experiment can be easily completed using a CCD camera, and an actuator to rotate around the cylindrical target. However with that method backscattering can be studied only by slightly deviating the cylinder from the vertical position (almost $<5^{\circ}$ ), so the camera will be out of the incident beam. Figure shows the experimental set-up.
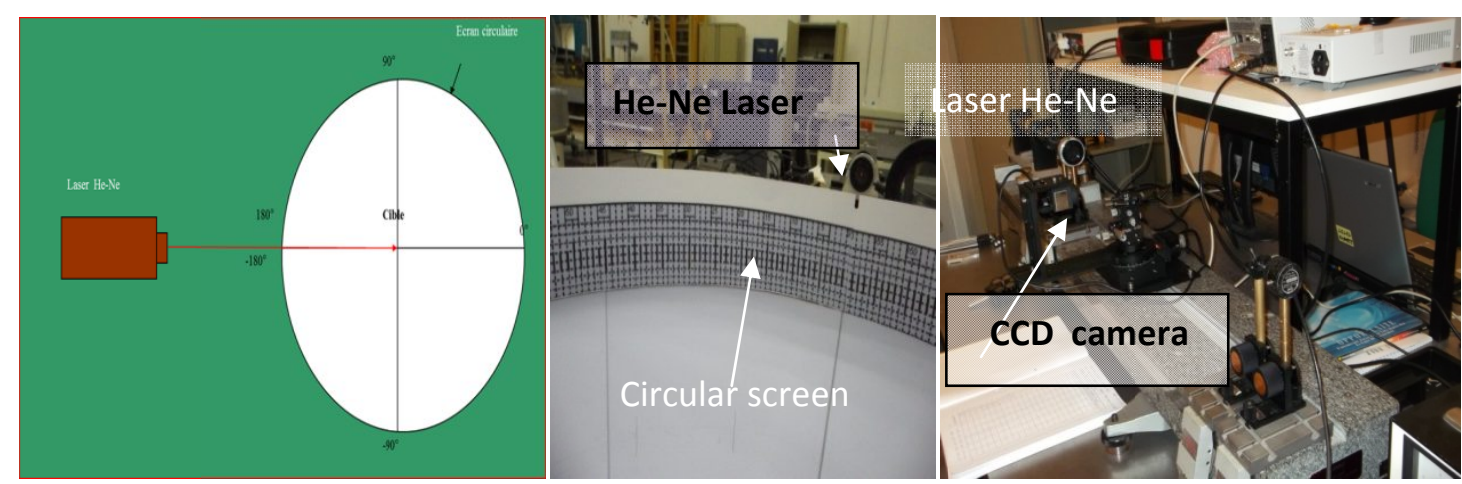

Figure 1: (a) schematic representation of the first experimental set-up, (b) a view of circular screen and the Laser source.

(c) a global view of the second experimental set-up using a CCD camera for intensity recording
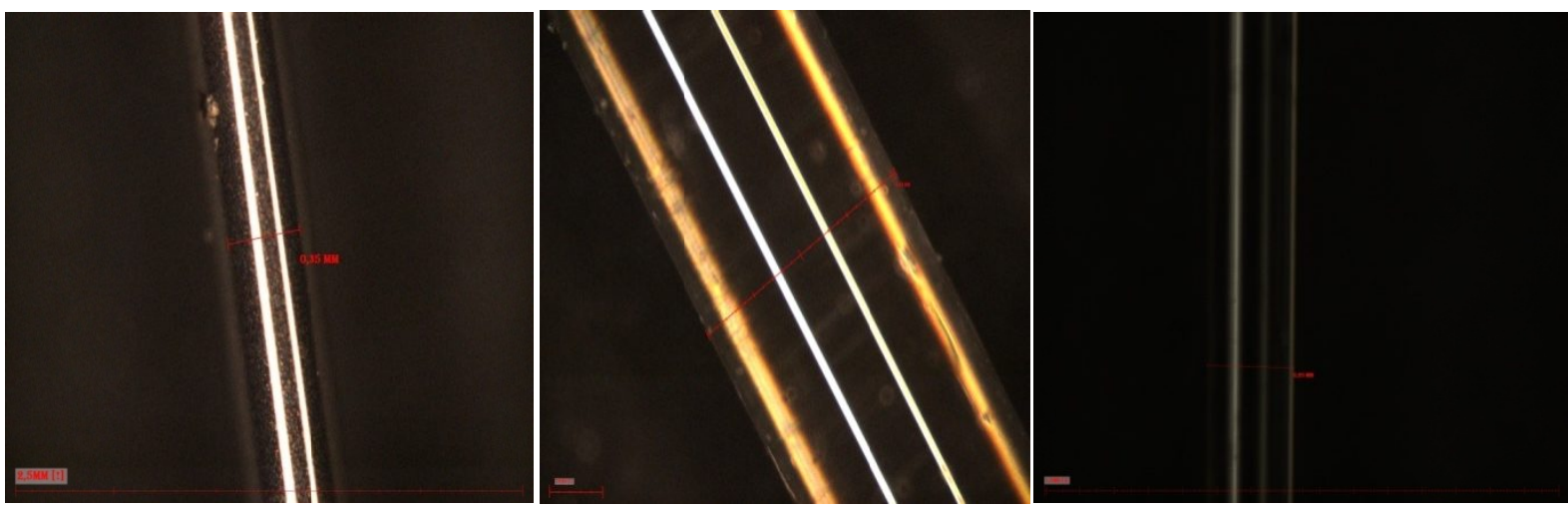

Figure 2:Micro-photograph of the samples used for the experiments: from left to right metallic cylinder (Nickel), total diameter $0.35 \mathrm{~mm}$. Low-loss dielectric cylinder (fused silica), diameter $2.03 \mathrm{~mm}$. High-loss cylinder (silicon monoxide) diameter $0.30 \mathrm{~mm}$. 


\section{RESULTS}

\subsection{Absorbing cylinders}

The material used for simulation and experiment is silicon monoxide. The far field intensity shows both in simulation (figure 6a: curves in yellow and red respectively) and experiments (figure3), a rapid decrease in the intensity profile in the vicinity of the forward direction, diffraction are quite noticeable both in simulation and experiments, but with a shift in their position (according to the state of light polarization), this lead to an oscillatory behavior of the linear polarization distribution (figure6b: curve in red). The experiment is unable to detect a noticeable intensity beyond $40^{\circ}$ degrees (figure3). The simulation performed shows that the intensity in that range is not zero, but very low. As an example for size parameter of 5000, the ratio of the zero order diffraction peak and the backscattered intensity is of $1 / 200$ in value. It also shows that the Brewster angle appears at the predicted values by the Fresnel model with a strong polarization $(89 \%$ for $\mathrm{x}=5000$ ), at that angle (incident angle $63.02^{\circ}$, corresponding to a scattering angle of $53.96^{\circ}$ )(figure $6 \mathrm{~b}$ : curve in red). As the size parameter decrease simulation shows that the scattering regime also changes, the intensity distribution tends to become uniform. When the size parameter reach values below 10, the diffraction regime tends to disappear and distribution becomes uniform (size parameter: 0.1 or lower), with a stronger fading of orthogonal polarization with respect to the cylinder axis. This lead to a uniform and appreciable linear polarization distribution: $38.3 \%$ (figure $7 \mathrm{~b}$ : red)

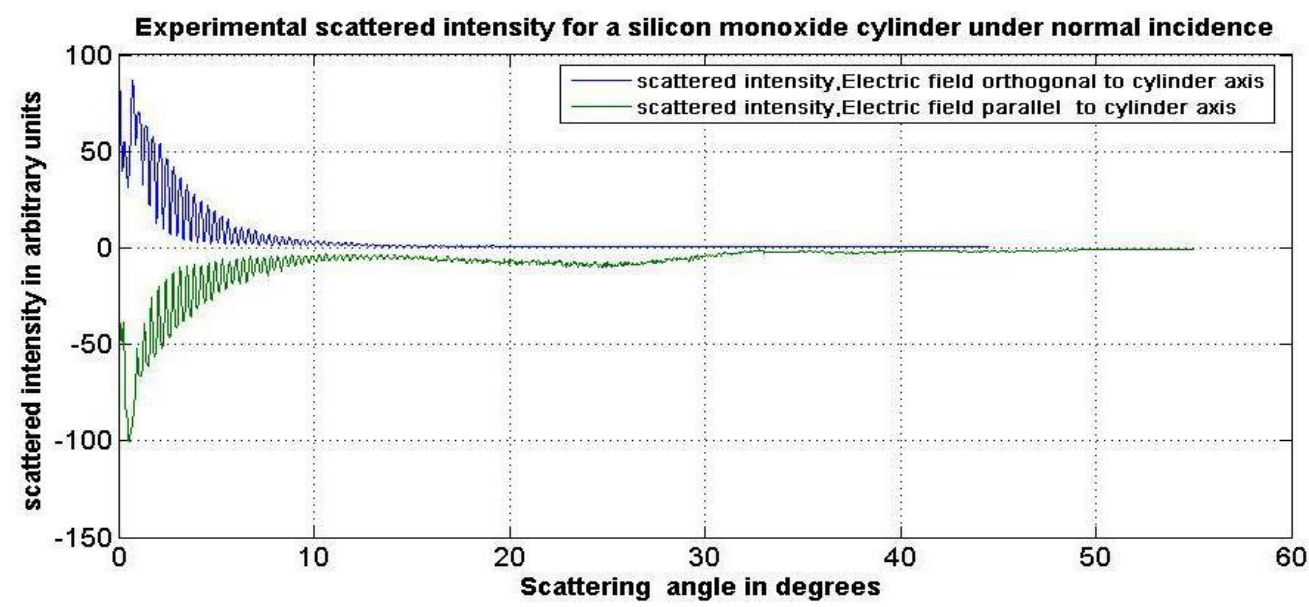

Figure 3: Experimental scattered intensity distribution for a silicon monoxide cylinder under normal incidence.

\subsection{Metallic cylinder}

The material used for both simulation and experiments is Nickel. The far field intensity as in the preceding case shows both in simulation (figure 6a: curves in dark green and black respectively) and experiments (figure 4a and 4b), a rapid decrease in the intensity profile in the vicinity of the forward direction, diffraction is quite noticeable both in simulation and experiments, but with a slight difference in the maxima separation for the same size parameter, narrower than for the absorbing cylinder. This mean a systematic overestimation of the diameter using Fraunhoffer approximation, some theoretical studies tend to prove this behavior ${ }^{(7)}$. 
The experiment shows a quite noticeable intensity distribution in the whole scattering range with a strong component in the backward region, this is due certainly to the strong reflectivity of metals in the optical range. A minimum appears in the intensity distribution at an angle corresponding to that of Brewster for the material, a comparison is made with the Fresnel model for both polarizations. While the Lorentz-Mie model shows that the Fresnel model is a good approximation for large cylinders, especially for studying the distribution of linear polarization. As the size parameter decrease the scattering regime also change tending to become, as in the preceding case uniform. But the fading of one the polarization is more important than that of the absorbing cylinder. The distribution becomes uniform for size parameters equals or lowers than one, reaching value of $70 \%$ ( figure...). Nano sized metallic cylinders are good polarizer.

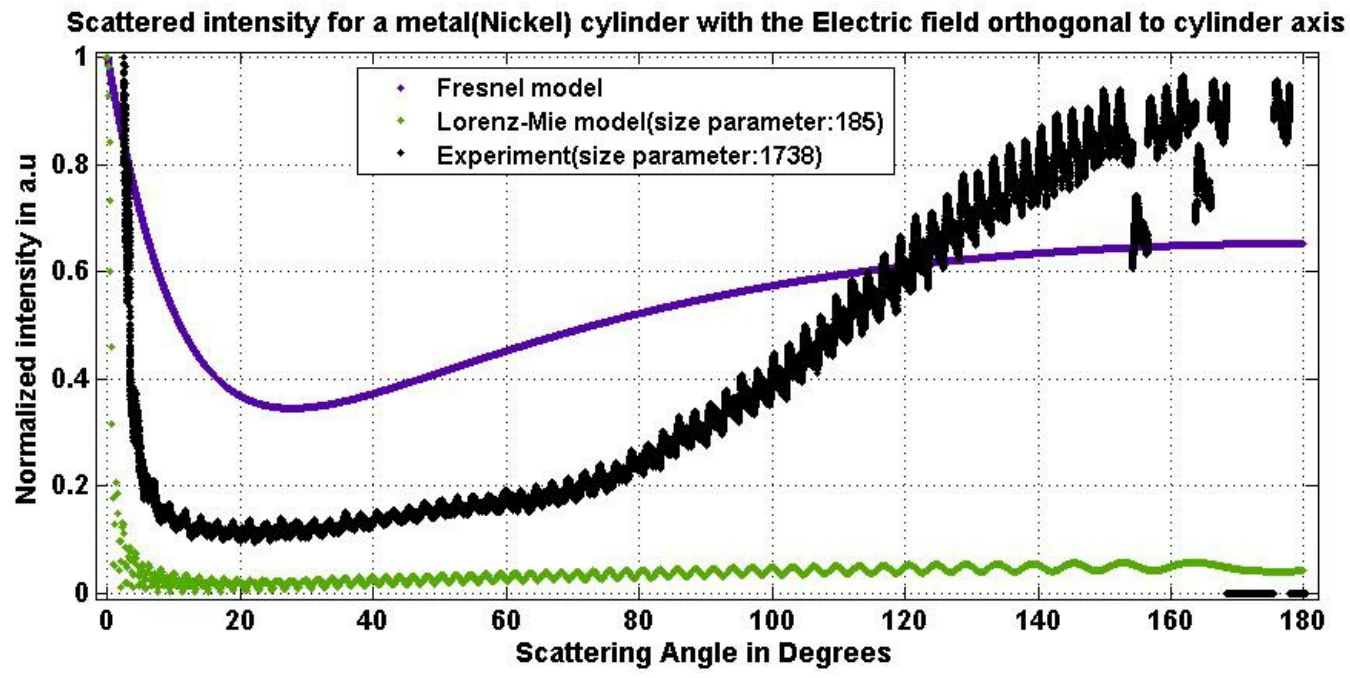

Figure.4a: Experimental scattered intensity distribution for a Nickel under normal incidence (black), electric field orthogonal to cylinder axis. Comparison with Fresnel model (purple), Lorentz-Mie model (green) with a different size parameter to illustrate its influence in the scattering regime.

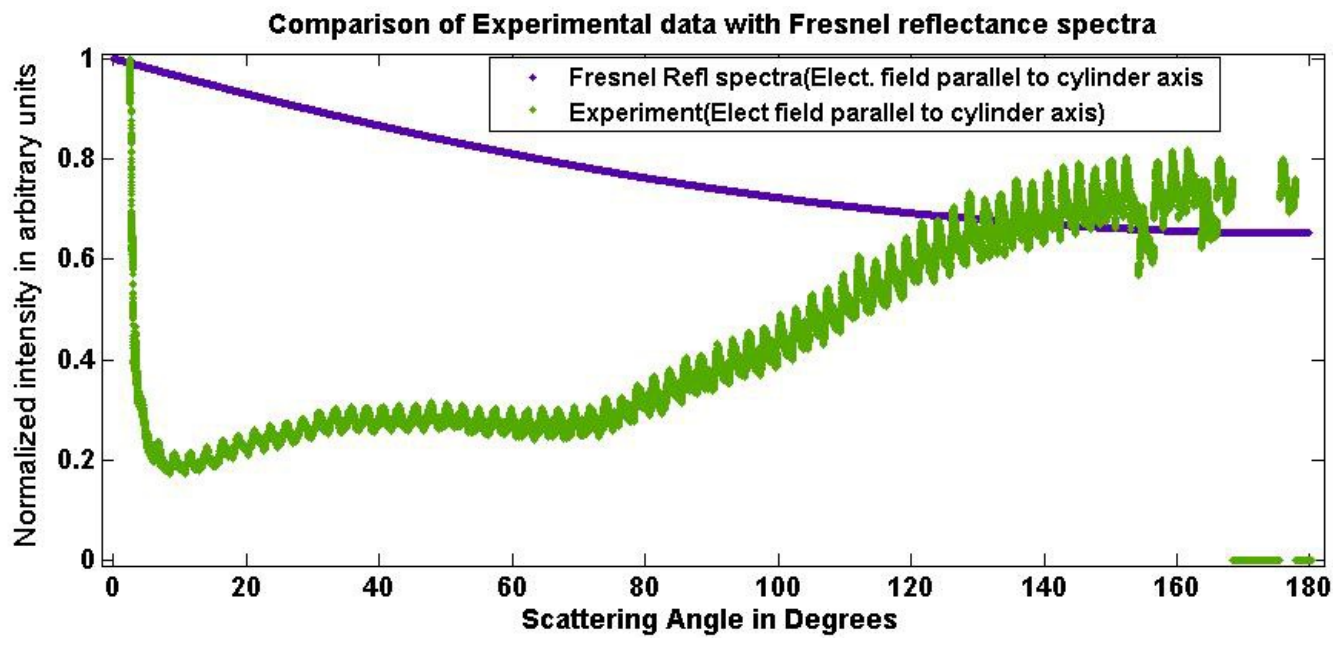

Figure.4b: Experimental scattered intensity distribution for a Nickel cylinder under normal incidence (green), electric field parallel to cylinder axis. Comparison with Fresnel model (purple). 


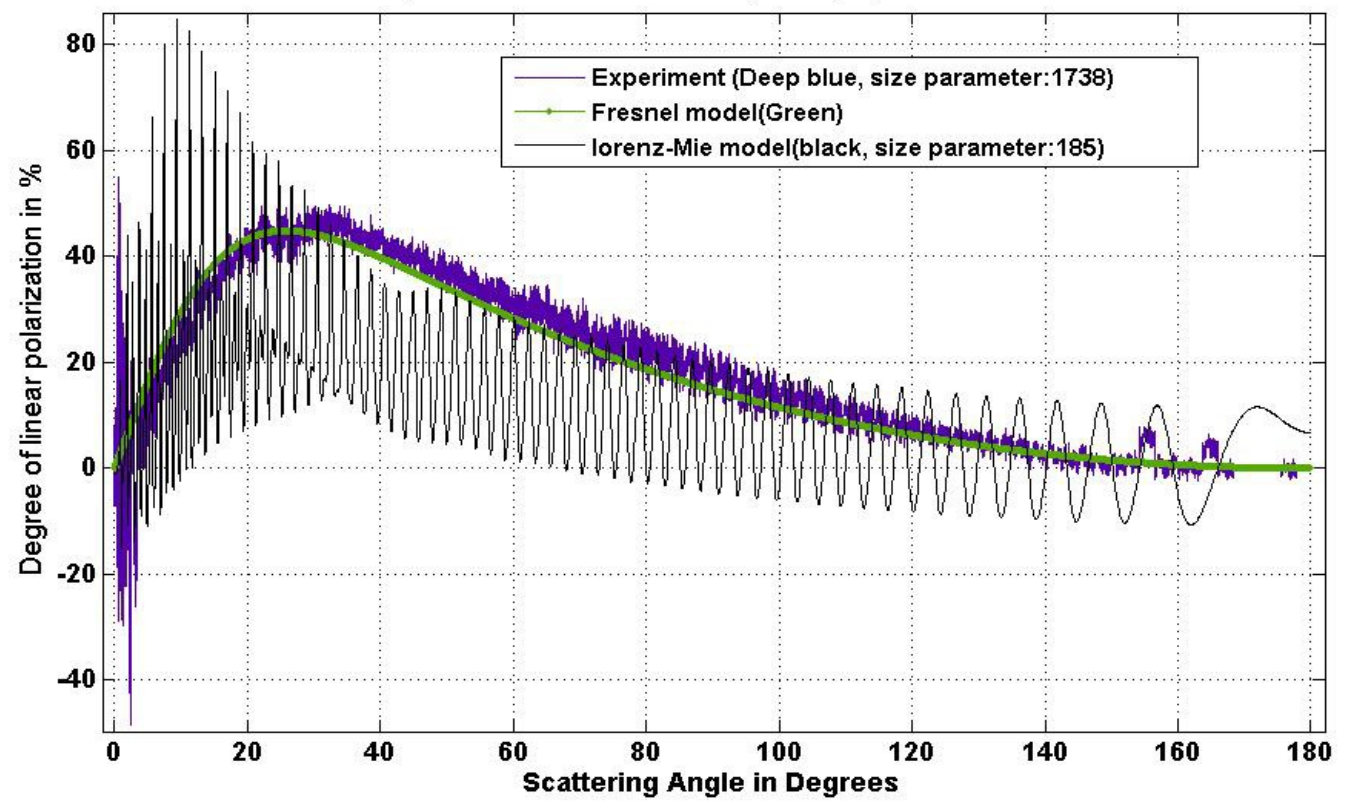

Figure 4c: experimental distribution of linear polarization of the Nickel cylinder under normal incidence (purple), comparison with the Fresnel model (green), Lorenz-Mie model for a different size parameter (185).

\subsection{Low loss dielectric cylinder}

The material used is fused silica. The far-field intensity distribution is quite different than in the preceding cases. The intensity distribution does not show a rapid decrease around the zero order peaks, both in simulation (figure 6a: curve in light green and deep blue) and experiment (figure5a). The maxima separation is not uniform, and than get wider with the increasing scattering angle. The Frauhnhoffer approximation is unsuited to describe this "anomalous diffraction pattern", and lead to an important relative error while trying to characterize particle diameter by that method, almost $>10 \%$. This is certainly due to other effects such as transmission through the interface that is superimposed to the diffraction pattern. Moreover, in the forward direction, the simulation shows a single peak, while the experiment shows interference patterns in the region of geometrical section (figure 5b). In the backward direction (scattering angle above $90^{\circ}$ ), rainbows signals (primary rainbow, surnumerary bows) can be seen in position predicted by different theories of the rainbows ${ }^{(8)}{ }^{(9)}$. Simulation and experiments show that the rainbow signal is visible only when the polarization is parallel to cylinder axis; leading to a strong polarization in the vicinity of that angle (figure 5c). Simulation shows that the peak position is slightly variable with the size parameter, and for value lower than 100 , the signal is not distinguishable with the interference pattern around it. The backscattering is also rich in interference which distribution depends on the size parameter. As the parameter size decreases the intensity distribution tends to become uniform(figure 7a:curves in dark and light green) and for value equals to 1 or lower, the distribution gets, as for the preceding cases, uniform with the same behavior, with a degree of light polarization equals to $17 \%$ (figure $7 \mathrm{~b}$ : green). 


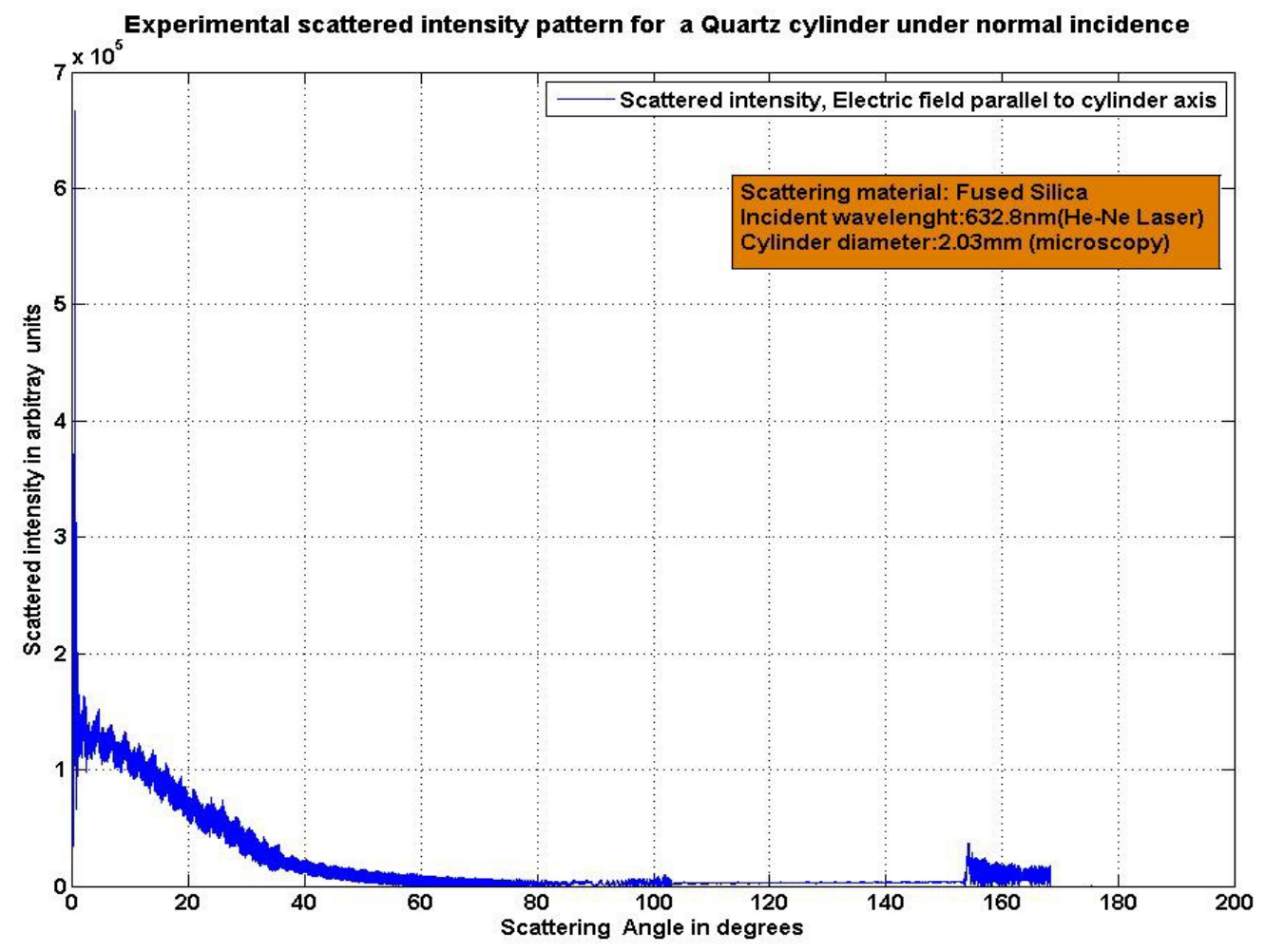

Figure.5a: Experimental scattered intensity distribution for a fused silica cylinder under normal incidence. Electric field parallel to cylinder axis. (Back scattering is not recorded above $169^{\circ}$ in that figure)
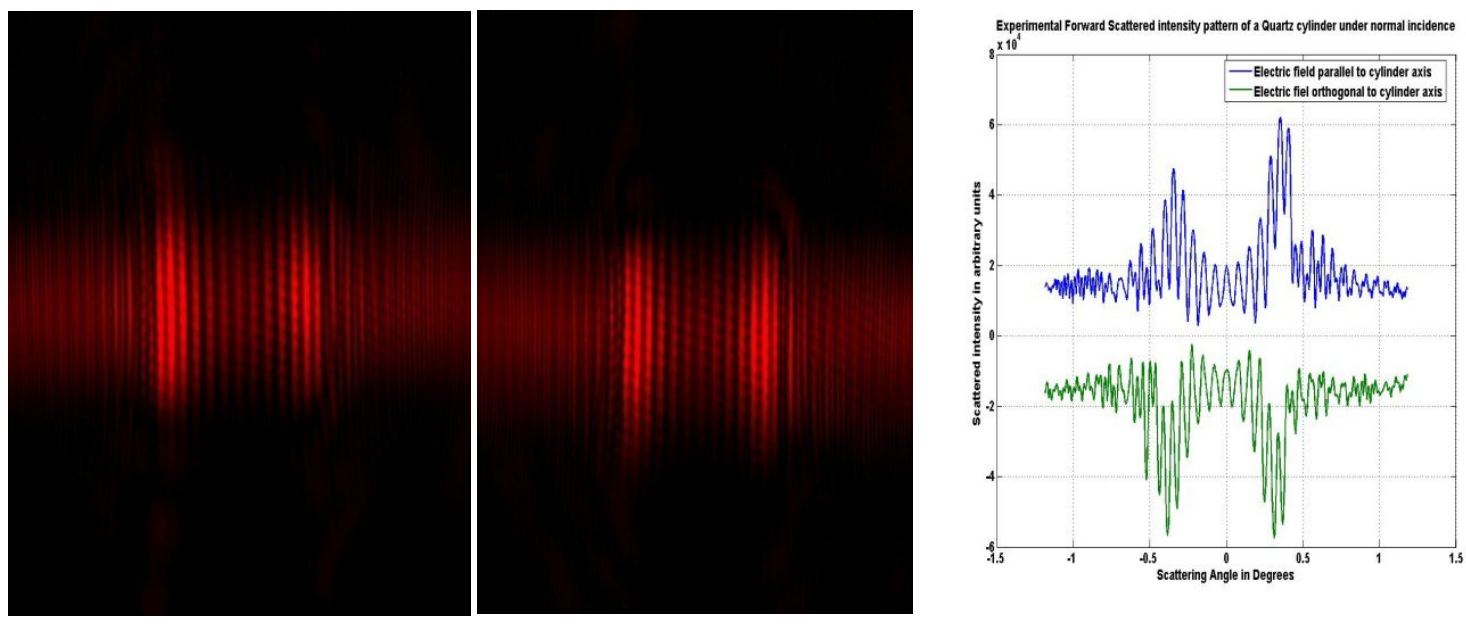

Figure 5b: interference pattern in the forward direction, in the geometrical section. Left (Electric field parallel to cylinder axis), centre (Electric field orthogonal to cylinder axis). Right: scattered pattern in the forward direction. Note the phase opposition between the polarizations. 


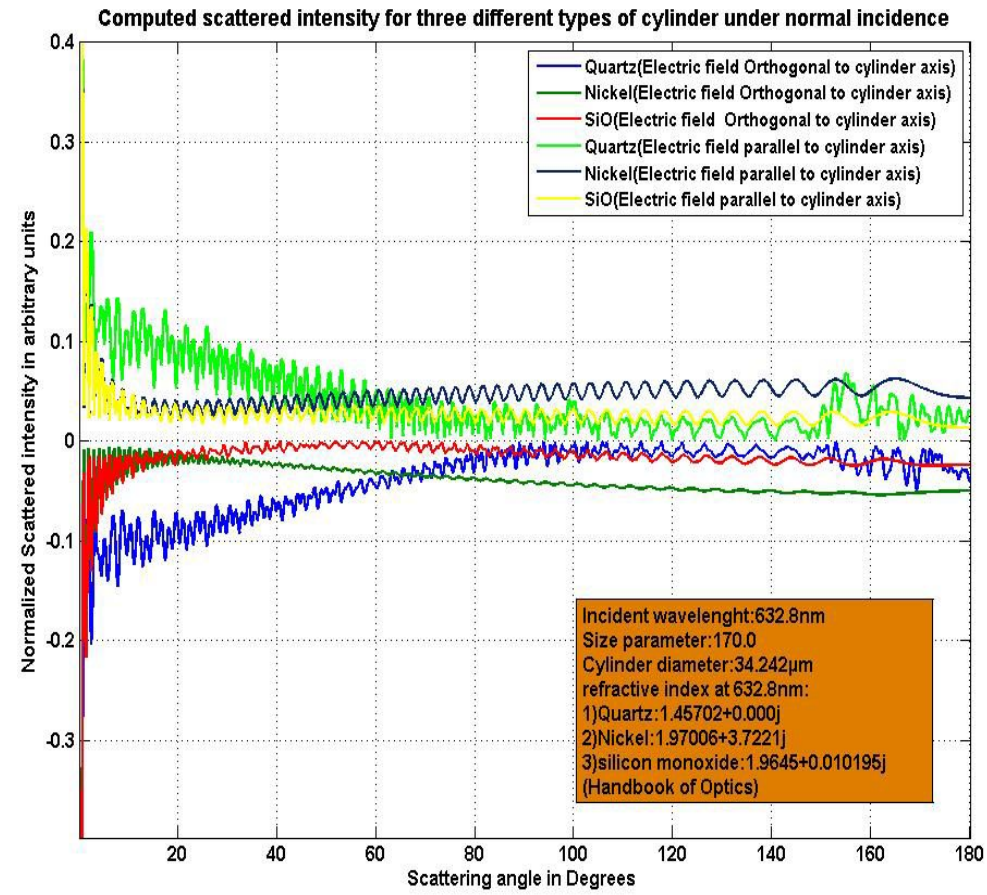

Figure 6a: compared computed scattered intensity for medium sized cylinders, of three different types: absorbing, metallic, and lowloss dielectric of same size parameter: 170. Under normal incidence.

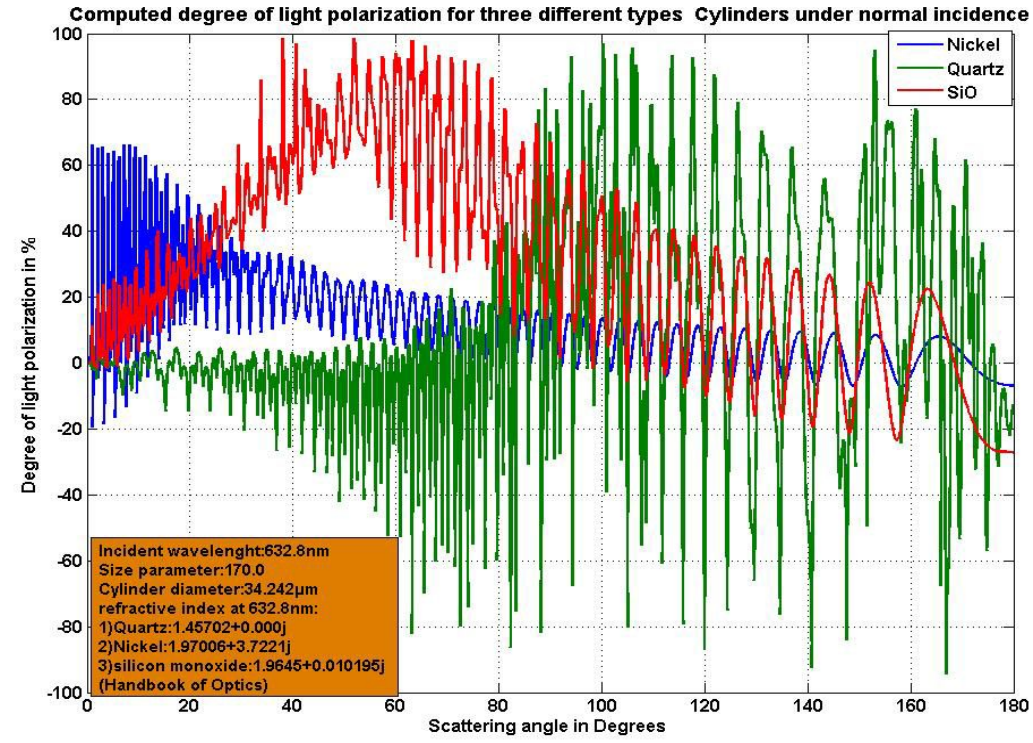

Figure 6b: associated distribution of linear polarization for the three types of cylinders under normal incidence 
Computed scattered intensity for three different types of cylinders under normal incidence

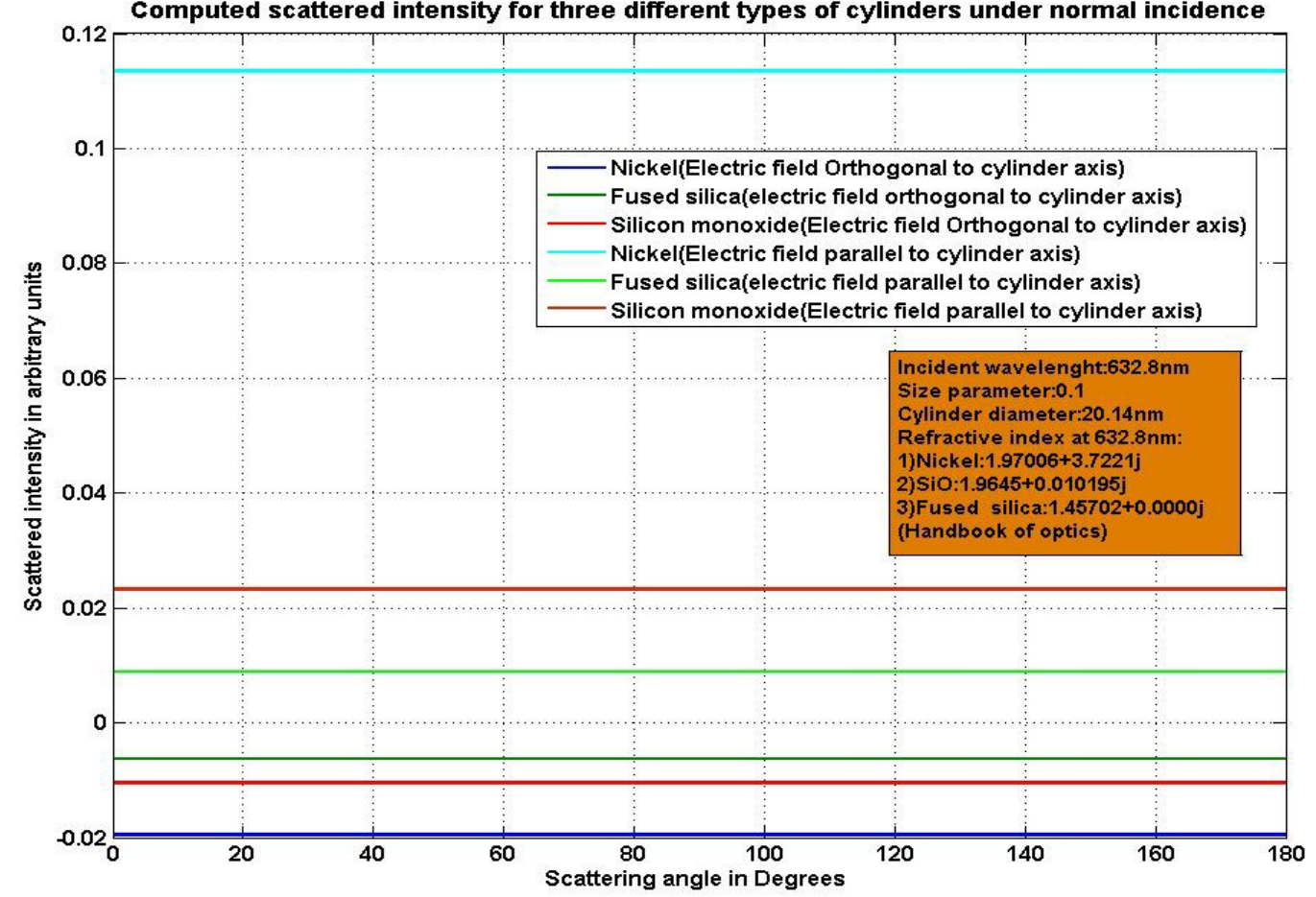

Figure 7a: comparative computed scattering pattern for sub wavelength cylinders (size parameter: 0.1 ) under normal incidence

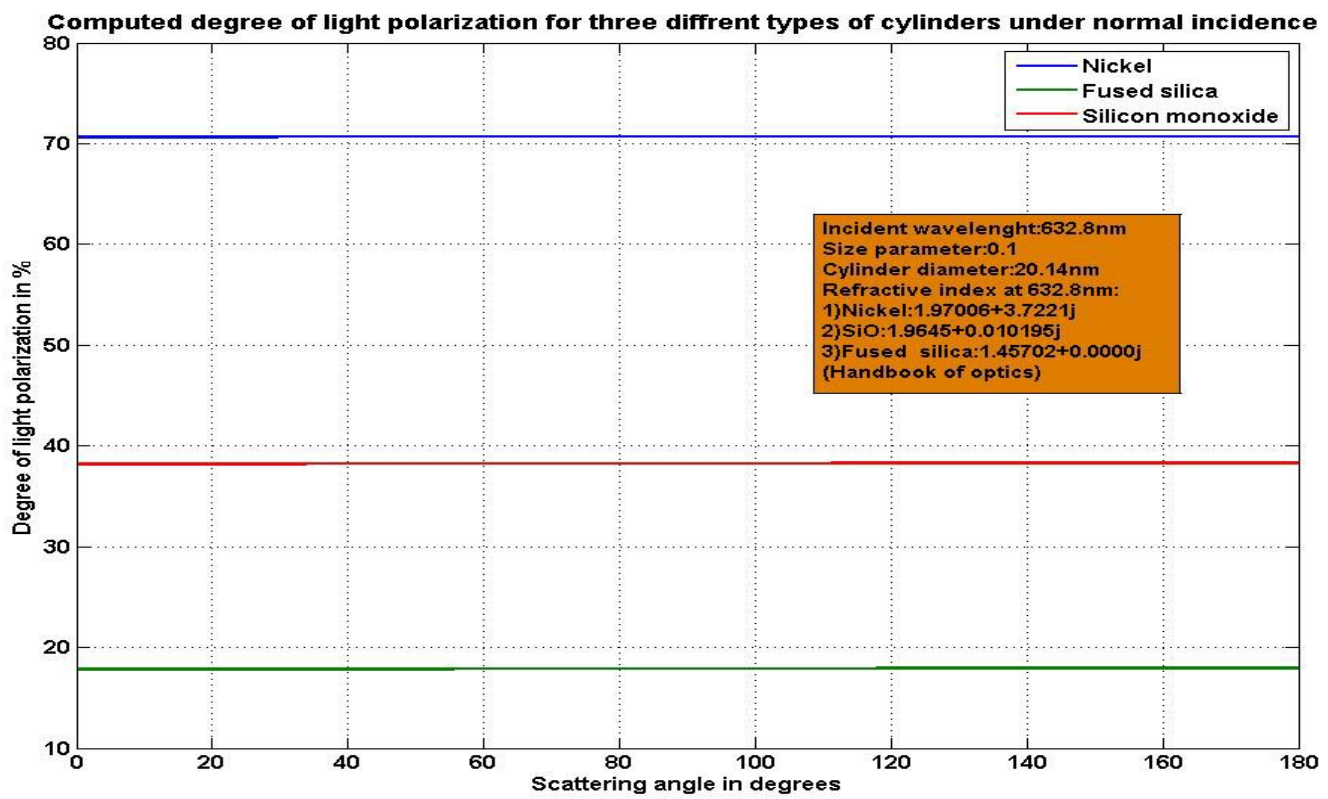

Figure $7 \mathrm{~b}$ : corresponding distribution of linear polarization, for the three types of cylinders. Size parameter: 0.1 


\section{CONCLUSION}

We have investigated through that simple laboratory practice the behavior of homogenous cylindrical particles, under normal light incidence. The study had pedagogical objectives using a double approach: experiment and simulations. Simulation codes are written on the basis of the Lorenz-Mie formalism. Both simulations and experiments show that the scattered regime depends both on the nature of the material and the size parameter. For the same material, a dependence on the state of light polarization is noticed. Simulations show, to some extents, the patterns displayed in experiments. With convergence for instance in rainbows (low-loss dielectric cylinders), and mismatch: forward peak in simulation versus interference patterns in experiments. At sub wavelength scale, simulations show that the distribution tends to becomes uniform, leading to a distribution of the intensity independent to the scattering angle (size parameter 0.1).

\section{REFERENCES}

[1] Jackson, J. D. [électrodynamique classique] . s.l. : DUNOD édition , (1995) .

[2] Vigneron, Jean Pol. Cylindrical Bragg mirrors of the male "Bolivian tarantula Pamphobeteus Antinous". Physical review, PACS numbers 42.66.-p,42.70Qs,42.81.-i,42.81.Qb., pp. 42.66-42.81. (January 2011)

[3] G. MIE., Ann Physik, p. 25377 (1908).

[4] Van de Hulst, H.C.[ Light scattering by small particles], chapter 9. New york : john Wiley \& sons, Inc. (1957).

[5] Kuo Nan Liou. "Electromagnetic scattering by arbitrarily oriented ice cylinders". [éd.] Optical society of America., J. Applied Optics, Vol. Volume 11, p. page 667, (March 1972).

[6] Wiscombe, W.J. "Improved Mie Scattering Algorithms". 19, Applied Optics, pp. 1505-1509, (1980).

[7] L. Miguel Sanchez Bréa, J.C.Martinez, E. Barnabeu."Effect of the refraction index in the diameter estimation of thin metallic wires". [éd.] SPIE. Bellingham,WA : s.n., , Proceedings of SPIE, Vol. 5858. doi:10.1117/12.612652 (2005).

[8] Airy, G.B "On the intensity of light in the neighborhood of a caustic". 6, Trans. Cambridge Phil. Soc., pp. 372-402. (1838).

[9] Beeck, J. Van. "Rainbow Phenomena: development of a Laser-based, non-intrusive technique for measuring Droplet size, temperature and velocity", . Eindhoven : Technische Universiteit Eindhoven, Thesis , (1997).

Proc. of SPIE Vol. $10452104522 \mathrm{U}-10$ 